\title{
Weighted Triangulations for Geometry Processing
}

\author{
Fernando de Goes \\ Caltech \\ and \\ Pooran Memari \\ CNRS-LTCI Telecom ParisTech \\ and \\ Patrick Mullen \\ Caltech \\ and \\ Mathieu Desbrun \\ Caltech
}

In this paper, we investigate the use of weighted triangulations as discrete, augmented approximations of surfaces for digital geometry processing. By incorporating a scalar weight per mesh vertex, we introduce a new notion of discrete metric that defines an orthogonal dual structure for arbitrary triangle meshes and thus extends weighted Delaunay triangulations to surface meshes. We also present alternative characterizations of this primal-dual structure (through combinations of angles, areas, and lengths) and, in the process, uncover closed-form expressions of mesh energies that were previously known in implicit form only. Finally, we demonstrate how weighted triangulations provide a faster and more robust approach to a series of geometry processing applications, including the generation of well-centered meshes, self-supporting surfaces, and sphere packing.

Categories and Subject Descriptors: I.3.5 [Computer Graphics]: Computational Geometry and Object Modeling-Curve, surface, solid, and object representations

Additional Key Words and Phrases: discrete differential geometry, discrete metric, weighted triangulations, orthogonal dual diagram.

ACM Reference Format:

Permission to make digital or hard copies of part or all of this work for personal or classroom use is granted without fee provided that copies are not made or distributed for profit or commercial advantage and that copies show this notice on the first page or initial screen of a display along with the full citation. Copyrights for components of this work owned by others than ACM must be honored. Abstracting with credit is permitted. To copy otherwise, to republish, to post on servers, to redistribute to lists, or to use any component of this work in other works requires prior specific permission and/or a fee. Permissions may be requested from Publications Dept., ACM, Inc., 2 Penn Plaza, Suite 701, New York, NY 10121-0701 USA, fax +1 (212) 869-0481, or permissions@acm.org.

(c) YYYY ACM 0730-0301/YYYY/14-ARTXXX $\$ 10.00$

DOI 10.1145/XXXXXXX.YYYYYYY

http://doi.acm.org/10.1145/XXXXXXX.YYYYYYY

\section{INTRODUCTION}

Triangle meshes are arguably the predominant discretization of surfaces in graphics, and by now there is a large body of literature on the theory and practice of simplicial meshes for computations. However, many geometry processing applications rely, overtly or covertly, on an orthogonal dual structure to the primal mesh. The use of such a dual structure is very application-dependent, with circumcentric and power duals being found, for instance, in physical simulation [Elcott et al. 2007; Batty et al. 2010], architecture modeling [Liu et al. 2013; de Goes et al. 2013] and parameterization [Mercat 2001; Jin et al. 2008]. While most of these results are limited to planar triangle meshes, little attention has been paid to exploring orthogonal duals for triangulated surface meshes.

In this paper, we advocate the use of orthogonal dual structures to enrich simplicial approximations of arbitrary surfaces. We introduce an extended definition of metric for these discrete surfaces with which one can not only measure length and area of simplices, but also length and area of dual elements. Our approach builds on the concept of weighted triangulations [Glickenstein 2005], i.e., triangle meshes equipped with a scalar weight per vertex, that generalize Delaunay/Voronoi and weighted-Delaunay/power dualities to arbitrary surface meshes (Fig. 1). While leveraging a number of theoretical results, our work is directly applicable and beneficial to common geometry processing tasks such as meshing.

\subsection{Related Work}

Our contributions relate to a number of research efforts and applications in computer graphics, computational geometry, and discrete mathematics. We briefly discuss the most relevant topics next.

Primal-Dual Meshes. While a discrete surface is often described as just a triangle mesh, an increasing number of methods make use of an orthogonal dual structure as well: this form of Poincaré duality [Munkres 1984] is valuable in modeling (e.g., for mesh parameterization [Mercat 2001]) and physical simulation (e.g., for fluid flows [Batty et al. 2010]) alike. Orthogonal dual meshes are most commonly constructed by connecting neighboring triangle circumcenters [Meyer et al. 2002]. However, this choice of dual mesh is only appropriate for so-called pairwise-Delaunay triangulations (see, e.g., [Dyer and Schaefer 2009; Hirani et al. 2013]), while most triangulations require combinatorial alterations for this dual to be well formed [Fisher et al. 2007]. This construc- 


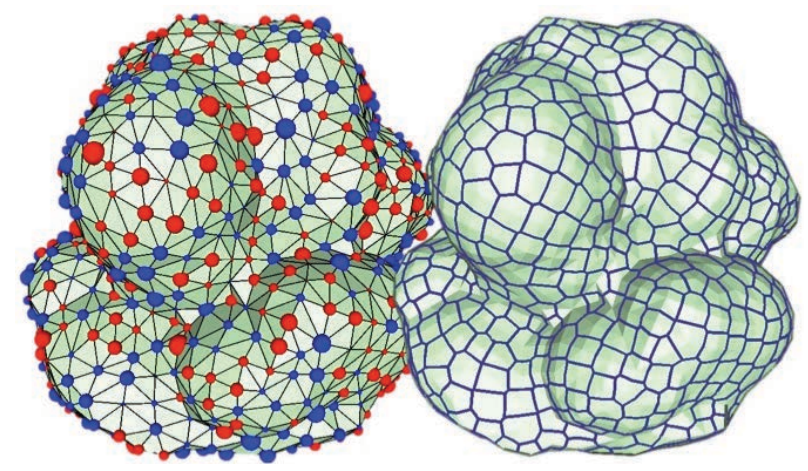

Fig. 1. Weighted Triangulation. Example of a triangle mesh equipped with vertex weights (left) displayed as spheres with squared radii corresponding to the weight magnitudes and colors according to their signs (red + , blue - ). The set of weights endows the triangulation with an orthogonal, non-circumcentric dual structure (right).

tion is thus often too restrictive for the demands of many graphics applications such as the generation of well-centered meshes [VanderZee et al. 2010; Mullen et al. 2011] or the construction of discrete Laplacian operators with only positive coefficients [Wardetzky et al. 2007; Vouga et al. 2012]. To overcome these issues, weighted triangulations [Glickenstein 2005] were introduced as an alternative with much greater flexibility in the location of dual vertices while maintaining primal-dual orthogonality. These orthogonal primal-dual structures have found recent adoption in geometry processing for mesh optimization [Mullen et al. 2011] and architectural design [Liu et al. 2013; de Goes et al. 2013]. In this paper, we present further theoretical foundations and computational methods required for the construction of weighted triangulations and their use in geometry processing.

Discrete Metric. Edge lengths are commonly used to define the discrete metric on triangle meshes: once edge lengths are given, measurements such as angles, areas, and Gaussian curvatures are easily computed [Regge 1961]. However, alternative representations of the discrete metric have also been shown effective in many geometry processing applications. Angles, for instance, are known to characterize the intrinsic geometry of a triangle mesh up to a global scaling. In fact, Rivin [Rivin 1994] even studied the use of angle sums (per vertex, or per edge) as a more concise encoding of the metric. His ideas were further explored and applied to circle patterns [Bobenko and Springborn 2003; Kharevych et al. 2006] and conformal equivalence [Springborn et al. 2008]: while the former derives the metric of a triangle mesh by assigning an angle-based curvature per edge, the latter determines the metric as a length cross ratio per edge and a curvature per vertex. More recently, Zeng et al. [2012] proved that the ubiquitous cotan formula [MacNeal 1949; Pinkall and Polthier 1993], used in the discrete Laplace-Beltrami operator, also determines all the edge lengths of a triangle mesh up to a global scaling and thus encodes its discrete metric. The notion of discrete metric for weighted triangulations has, comparatively, received far less attention. Our work presents several characterizations of the augmented metric resulting from the orthogonal primaldual structure of weighted triangulations.

Circle packing. Weighted triangulations are closely related to the circle packing problem, as each vertex weight can be interpreted as the squared radius of a vertex-centered circle. This problem goes back to an unpublished work by W. Thurston that presented circle packing as a discrete notion of conformal mapping [Thurston
1976]. Packing circles on triangle meshes has been extensively investigated in graphics. The assignment of circles per triangle, for instance, corresponds to the circle pattern problem for which convex functionals were introduced in [Rivin 1994; Bobenko and Springborn 2003; Bobenko et al. 2010] and applied to mesh parameterization [Kharevych et al. 2006]. Another example, used in graphics to compute geometric flows [Jin et al. 2008], is the Andreev-Thurston circle packing [Stephenson 2003; Chow and Luo 2003] which defines a family of vertex-centered circles such that the circles incident to any edge intersect. This idea was further extended to non-intersecting circles through inversive distance circle packing [Guo 2009; Yang et al. 2009; Luo 2010], while tangency of neighboring circles corresponds to sphere packing [Colin de Verdière 1991]. Schiftner et al. [2009] showed that sphere packing only exists for triangle meshes in which the incircles of neighboring triangles are also tangent. Our definition of the augmented metric of weighted triangulations encompasses (and helps put in perspective) all these variants of the circle packing problem on arbitrary triangulated surfaces. In particular, our methodology to construct orthogonal dual meshes provides a novel and reliable computational framework to generate sphere and incircle packing.

\subsection{Contributions and Overview}

We begin our exposition by introducing our geometric construction of weighted triangulations in Sec. 2. We then define an admissible set of discrete metrics for these weighted triangulations in Sec. 3. We detail in Sec. 4 how this augmented metric can be directly used to derive discrete differential operators, such as the LaplaceBeltrami operator, that retain important properties of their smooth counterparts. We then present in Sec. 5 three alternative representations of the metric of a weighted triangulation, tying together a large number of previous works. In Sec. 6, we use our definition of metric on weighted triangulations to design new algorithms for meshing: we first offer an alternative to [Liu et al. 2013] in order to construct self-supporting triangulations with constant relative mean curvature through a convex optimization (Sec. 6.1), we then extend the work of [Mullen et al. 2011] to generate well-centered surface meshes (Sec. 6.2), and finally we provide a new approach to sphere packing on surfaces that improves robustness compared to [Schiftner et al. 2009] (Sec. 6.3). We conclude in Sec. 7 with a discussion of open problems and future work.

\section{DISCRETIZING SURFACES}

In this section we describe the discretization of surfaces through weighted triangulations. We first set basic definitions about discrete surfaces and introduce the notion of orthogonal dual diagrams. We then present weighted triangulations and provide closed-form expressions that relate the construction of orthogonal dual diagrams to weighted triangle meshes.

\subsection{Primal Mesh}

We begin with the common definition of piecewise linear triangle meshes to approximate smooth surfaces.

DEFINITION 1. A primal mesh $T$ is a piecewise linear triangulation with vertices $V$, edges $E$, and faces $F$, defining a discrete orientable manifold surface of any topology in $\mathbb{R}^{3}$, with or without boundary.

For the remainder of the paper, we use single indices (e.g., $i$ ) to refer to vertices of $T$, pairs of indices (e.g., $i j$ ) for edges, and triples 
(e.g., $i j k$ ) for triangles. The positions of the vertices are denoted as $\mathbf{x}_{i}$, and an edge $i j$ can be seen as a $3 \mathrm{D}$ vector $\mathbf{e}_{i j}=\mathbf{x}_{j}-\mathbf{x}_{i}$. The length of edge $i j$ is denoted by $l_{i j}=\left|\mathbf{e}_{i j}\right| \in \mathbb{R}^{+}$, while $a_{i j k} \in \mathbb{R}^{+}$represents the area of triangle $i j k$. We use $\psi_{i}$ to refer to the piecewise linear basis function over $T$ for the vertex $i$ (i.e., $\left.\psi_{i}\left(\mathbf{x}_{j}\right)=\delta_{i j} \forall j \in V\right)$, with which nodal values can be linearly interpolated over $T$. Angles in $T$ are indexed by the triangle they live in, with the middle index referring to the originating vertex: $\alpha_{i j k}$ thus denotes the tip angle of triangle $i j k$ at vertex $j$ (Fig. 2, left). Additionally, we define $\theta_{i} \in \mathbb{R}^{+}$as the sum of all tip angles at vertex $i$, and $\phi_{i j} \in(0,2 \pi)$ as the sum of the (up to two) angles opposite to edge $i j$. Finally, we call $\Omega_{i}$ the set of simplices incident to vertex $i$.

\subsection{Dual Diagram}

We enrich the surface discretization by incorporating a dual diagram to the triangulation $T$, defined via the duality map $*$ that transforms a simplex of $T$ into its dual element [Munkres 1984].

DEFINITION 2. The dual diagram of $T$ associates every triangle ijk to a dual vertex $* i j k$, every edge ij to a dual edge $* i j$ connecting the dual vertices of its adjacent triangles, and every vertex $i$ to the dual cell $* i$ formed by the cycle of its neighboring dual edges.

Note that this notion of a dual diagram is purely combinatorial since the actual locations of dual vertices (and thus of the edges) are not given yet. We now focus on the case where dual vertices are placed within the plane of their associated triangles.

Definition 3. A dual diagram is denoted intrinsic to $T$ if every dual vertex $* i j k$ is located at a position $\mathbf{c}_{i j k}$ restricted to the plane supporting triangle ijk.

From this definition any dual edge $* i j$ of $T$ can be constructed by isometrically unfolding the pair of adjacent triangles $i j k$ and $i m j$ into the plane, and joining the dual vertices $\mathbf{c}_{i j k}$ and $\mathbf{c}_{i m j}$ with a straight path (Fig. 2, right). We hence refer to such dual edges as intrinsically straight segments. We finally introduce an orthogonality condition between primal and dual edges.

Definition 4. An orthogonal dual diagram is an intrinsic dual diagram in which the supporting line of each isometrically unfolded dual edge is orthogonal to its respective primal edge.

A common example of an orthogonal dual diagram consists of associating each dual vertex $* i j k$ to its triangle circumcenter, i.e., the point in the plane of the triangle that is equidistant from the three vertices $\mathbf{x}_{i}, \mathbf{x}_{j}$, and $\mathbf{x}_{k}$. In the case of Delaunay triangulations, these circumcentric dual diagrams correspond to Voronoi
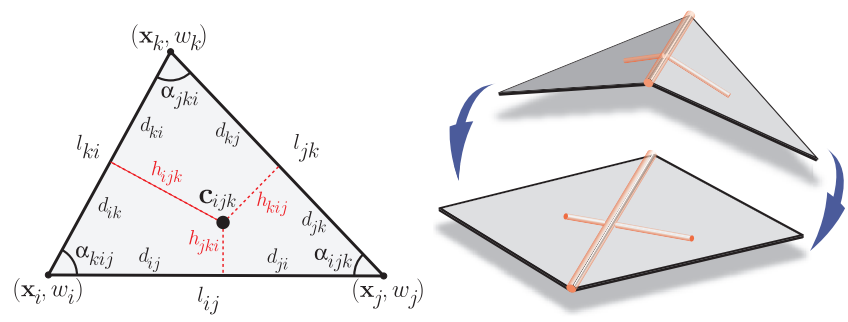

Fig. 2. Notations. (left) The various measurements and positions of the elements of a triangle are labeled based on the indices of its vertices as indicated above; (right) for every pair of adjacent triangles, the primal edge and its dual edge are orthogonal in the hinge map, i.e., when the pair is isometrically unfolded in the plane. diagrams. Hereafter we use $\mathbf{c}_{i j k}^{\diamond}$ to denote the circumcenter of triangle $i j k$.

\subsection{Weighted Triangulations}

Our work focuses on weighted triangulations [Glickenstein 2005], a family of triangulations that provides explicit control over the construction of orthogonal dual diagrams.

DEFINITION 5. A weighted triangulation $T^{w}$ is a primal mesh $T$ with an additional set of real numbers $w=\left\{w_{i}\right\}$, called weights, assigned to the vertices of $T$.

From these additional weights, we define the position $\mathbf{c}_{i j k}$ of the dual vertex $* i j k$ as the displacement of the circumcenter $\mathbf{c}_{i j k}^{\diamond}$ by (half) the local gradient of the linear function defined by the vertex values $w_{i}, w_{j}$, and $w_{k}$.

DEFINITION 6. The location $\mathbf{c}_{i j k}$ of the dual vertex $* i j k$ in $T^{w}$ is defined as:

$$
\begin{aligned}
\mathbf{c}_{i j k} & =\mathbf{c}_{i j k}^{\diamond}-\frac{1}{2}(\nabla w)_{i j k} \\
& =\mathbf{c}_{i j k}^{\diamond}-\frac{1}{2}\left(w_{i} \nabla \psi_{i}+w_{j} \nabla \psi_{j}+w_{k} \nabla \psi_{k}\right) .
\end{aligned}
$$

Note that the displacement per triangle occurs within the triangle's supporting plane, and thus the dual diagram of any $T^{w}$ is by construction intrinsic to the primal mesh $T$. Moreover, a gradient vector is unchanged if one adds a constant to all weights; thus, weights add $|V|-1$ degrees of freedom to a primal mesh $T$. It is worth pointing out that this construction does not necessarily result in dual nodes being inside their own triangle. This notion of "off-centered" dual is well documented in the specific case of circumcentric dual for obtuse triangles [Meyer et al. 2002], and our construction is no different.

We can further show that the dual structure of any $T^{w}$ is an orthogonal diagram.

Proposition 1. A weighted triangulation $T^{w}$ defines an orthogonal dual diagram.

Proof. Based on Eq. (1), one can check analytically that both vertices of the dual edge $* i j$ orthogonally project onto the supporting line of the primal edge $i j$ at the same point:

$$
\mathbf{c}_{i j}=\mathbf{x}_{i}+\left(\frac{l_{i j}^{2}+w_{i}-w_{j}}{2 l_{i j}^{2}}\right) \mathbf{e}_{i j} .
$$

As a result, the supporting line of $* i j$ passes through $\mathbf{c}_{i j}$ and is indeed orthogonal to the primal edge.

With the dual diagram in place, we can introduce explicit formulae for the signed measures of the elements in an orthogonal dual diagram. We first define the signed distance between a vertex $\mathbf{x}_{i}$ and point $\mathbf{c}_{i j}$ as $d_{i j}$, with a positive sign if $\left(\mathbf{x}_{i}-\mathbf{c}_{i j}\right) \cdot\left(\mathbf{x}_{i}-\mathbf{x}_{j}\right) \geq 0$, and negative otherwise; i.e.,

$$
d_{i j}=\frac{l_{i j}^{2}+w_{i}-w_{j}}{2 l_{i j}}, \quad d_{j i}=\frac{l_{i j}^{2}+w_{j}-w_{i}}{2 l_{i j}} .
$$

Note that $d_{i j}+d_{j i}=l_{i j}$. We also define the "height" distance $h_{j k i}$ to be the signed distance between $\mathbf{c}_{i j k}$ and $\mathbf{c}_{i j}$, with a positive sign if the triangle $\left(\mathbf{c}_{i j k}, \mathbf{x}_{i}, \mathbf{x}_{j}\right)$ has the same orientation as the triangle $\left(\mathbf{x}_{i}, \mathbf{x}_{j}, \mathbf{x}_{k}\right)$, and negative otherwise; i.e.,

$$
h_{j k i}=\frac{l_{i j} \cot \alpha_{j k i}}{2}+\frac{\cot \alpha_{k i j}}{2 l_{i j}}\left(w_{j}-w_{k}\right)+\frac{\cot \alpha_{i j k}}{2 l_{i j}}\left(w_{i}-w_{k}\right) \text {. }
$$


This height definition allows us to define a signed length $l_{i j}^{*}$ of the dual edge $* i j$ associated with a primal edge $i j$ common to $i j k$ and $i m j$ as:

$$
l_{i j}^{*}=h_{j k i}+h_{i m j} .
$$

Note that this choice of signs matches the traditional sign convention for circumcentric duals (see [Hirani et al. 2013] for a recent exposition). Finally one can compute the signed area $a_{* i}$ of a dual cell $* i$ as the sum of the signed areas of triangles formed by vertex $i$ and each surrounding dual edge, resulting in:

$$
a_{* i}=\frac{1}{2} \sum_{j \in \Omega_{i}} d_{i j} l_{i j}^{*} .
$$

\subsection{Discussion}

Our embedding of dual vertices in $\mathbb{R}^{3}$ through displacements of circumcenters links to previous work in many aspects. By rewriting the gradient of basis function $\psi_{i}$ in triangle $i j k$ as $\mathbf{e}_{j k}^{\perp} /\left(2 a_{i j k}\right)$ (where $\perp$ denotes in-plane ccw rotation by $\pi / 2$ ) and using the geometric expression of the circumcenter $\mathbf{c}_{i j k}^{\diamond}$ given in [Alliez et al. 2005], we can reexpress the dual position as:

$$
\mathbf{c}_{i j k}=\mathbf{x}_{i}+\frac{1}{4 a_{i j k}} \sum_{p \in\{j, k\}}\left(\left(\left|\mathbf{x}_{i}-\mathbf{x}_{p}\right|^{2}+w_{i}-w_{p}\right) \mathbf{e}_{i p}^{\perp}\right) .
$$

This is the expression given in [Mullen et al. 2011] to define dual vertices as weighted circumcenters. We thus conclude that our notion of weighted triangulation matches their work, as well as the work of [Glickenstein 2005] that they built upon. Our new geometric interpretation of the dual embedding will be, however, crucial in the remainder of this paper.

Weighted circumcenters define a notion of triangle center that is equidistant to the triangle vertices based on an altered Euclidian distance, known as power distance. These centers are closely related to weighted Delaunay triangulations, an extension of Delaunay meshes that triangulates points enriched with a weight assignment [Aurenhammer 1987]. As in the Delaunay/Voronoi duality, weighted Delaunay triangulations also define an orthogonal dual diagram with positive dual lengths $\left(l_{i j}^{*} \geq 0\right)$, named power diagram. The duality of weighted Delaunay triangulations and power diagrams has found recent applications in graphics including mesh optimization [Mullen et al. 2011], displacement interpolation [Mérigot 2011], blue noise sampling [de Goes et al. 2012], and architecture modeling [Liu et al. 2013; de Goes et al. 2013].

Weighted triangulations are, instead, a generalization of weighted Delaunay triangulations that decouples mesh connectivity from weight assignment. In fact, any weighted triangulation can be converted to a weighted Delaunay triangulation through a series of edge flips to enforce positive dual lengths [Aurenhammer 1987]. Glickenstein [2005] showed that, for simply connected domains, the family of weighted triangulations includes all possible pairs of orthogonal primal and dual meshes. The case of meshes with arbitrary topology was recently addressed in [de Goes et al. 2013], where additional topological degrees of freedom were identified to fully span the space of orthogonal dual diagrams. While we leverage these previous results, our work explores the use of weighted triangulations to define an augmented notion of metric for discrete surfaces, as we described next.

\section{METRIC OF WEIGHTED TRIANGULATIONS}

The metric of a smooth surface defines an inner product between tangent vectors, thus allowing to measure length, area, and Gaus- sian curvature on the surface. On a triangle mesh, this notion of metric needs to be properly discretized in order to provide the same set of measurements. We now revisit the definition of metric on discrete surfaces and present validity conditions for the case of weighted triangulations.

\subsection{Primal Metric}

A triangulation represents a piecewise flat surface, hence the metric of the primal mesh alone is piecewise Euclidian. Arguably the most common representation of this metric is through $|E|$ edge lengths, used in many scientific contexts, including early work in numerical relativity [Regge 1961]. In order to define valid triangles, the lengths of a mesh must be positive and satisfy triangle inequalities.

DEFINITION 7. A primal metric in $T^{w}$ is a set of edge lengths $\left\{l_{i j}\right\}$ such that

$$
\begin{array}{ll}
\forall i j \in E: \quad & l_{i j}>0, \\
\forall i j k \in F: & l_{i j}+l_{j k}-l_{k i}>0, \\
& l_{j k}+l_{k i}-l_{i j}>0, \\
& l_{k i}+l_{i j}-l_{j k}>0 .
\end{array}
$$

We hereafter denote by $\mathbb{L}$ the set of primal metrics.

\subsection{Dual Metric}

Our weight-based construction of a dual brings about the need to measure dual elements as well, for which the primal lengths alone do not suffice. With lengths and weights, one can express the measure of each dual edge and cell as described in Sec 2.3. The dual structure generated by the weights is, however, unique to the discrete case since the distinction between primal and dual vanishes in the smooth limit. We thus define a dual metric as a restricted set of $|V|-1$ weights whose range vanishes as the mesh is appropriately refined (see, e.g., [Hildebrandt et al. 2006]) towards a smooth surface.

DEFINITION 8. A dual metric in $T^{w}$ is a set of vertex weight $\left\{w_{i}\right\}$ such that

$$
\forall i j \in E: \quad\left|w_{i}-w_{j}\right| \leq l_{i j}^{2} .
$$

Using Eq. (2), we can reexpress this condition as $d_{i j} \geq 0$. Geometrically, this means that the supporting line of each (isometrically unfolded) dual edge of $T^{w}$ intersects the interior of its associated primal edge. The dual structure is therefore constrained to "follow" the primal structure as it converges to a smooth surface under refinement. Note, however, that this condition does not enforce positivity of the dual edge lengths.

\subsection{Augmented metric}

Once primal and dual metrics are known, one can measure any mesh element of a weighted triangulation $T^{w}$, be it primal or dual. We thus refer to the pair $(l, w)$ of edge lengths $l=\left\{l_{i j}\right\}$ (determining the primal mesh) and weights $w=\left\{w_{i}\right\}$ (defining the orthogonal dual mesh) as the augmented metric of $T^{w}$.

DEFINITION 9. We define $\mathbb{L}^{\text {W }}$ as the set of augmented metrics (i.e., with lengths and weights satisfying Definitions 7 and 8), with a total of $|E|+|V|-1$ degrees of freedom.

\section{DISCRETE DIFFERENTIAL OPERATORS}

Equipped with an augmented metric we can derive discrete operators, useful in geometry processing, which make use of both primal 
and dual meshes. Discrete Exterior Calculus (DEC) is a particularly convenient language to easily deduce such operators [Bossavit 1998; Desbrun et al. 2007; Grady and Polimeni 2010].

\subsection{DEC on weighted triangulations}

In exterior calculus, computations on a smooth surface often involve the Hodge star operator, which is a metric-dependent map from differential $p$-forms to $(2-p)$-forms $(p=0,1,2)$, and the exterior derivative that maps $p$-forms to $(p+1)$-forms [Abraham et al. 1988]. In DEC, the primal-dual orthogonality is exploited to define a discrete Hodge star operator as a diagonal matrix containing ratios between the volumes of dual and primal elements. This definition remains directly applicable to weighted triangulations, leading to the following three expressions for discrete stars operating on primal two-, one-, and zero-forms:

$$
\begin{gathered}
\text { Primal two-forms: } \star_{2}=\operatorname{diag}\left(\left\{\star_{i j k}\right\}_{i j k \in F}\right) \text {, with } \\
\star_{i j k}=1 / a_{i j k} . \\
\text { Primal one-forms: } \star_{1}=\operatorname{diag}\left(\left\{\star_{i j}\right\}_{i j \in E}\right) \text {, with } \\
\star_{i j}=l_{i j}^{*} / l_{i j} . \\
\text { Primal zero-forms: } \star_{0}=\operatorname{diag}\left(\left\{\star_{i}\right\}_{i \in V}\right) \text {, with } \\
\star_{i}=a_{* i} .
\end{gathered}
$$

Observe that since the dual structure for constant weights corresponds to the circumcentric dual, the Hodge star for one-forms reduces to the well known cotan formula in this case [MacNeal 1949]. We will use $\star_{i j}^{\diamond}$ to denote this "unweighted" star, i.e., for an edge $i j$ between triangles $i j k$ and $i m j$,

$$
\left.\star_{i j}^{\diamond} \equiv \star_{i j}\right|_{w=\text { const }}=\frac{1}{2}\left(\cot \alpha_{j k i}+\cot \alpha_{i m j}\right) .
$$

Based on Eqs. (3) and (4), the weighted version of the Hodge star for one-forms is therefore:

$$
\begin{aligned}
\star_{i j}=\star_{i j}^{\diamond} & +\frac{\cot \alpha_{k j i}}{2 l_{i j}^{2}}\left(w_{i}-w_{k}\right)+\frac{\cot \alpha_{j i k}}{2 l_{i j}^{2}}\left(w_{j}-w_{k}\right) \\
& +\frac{\cot \alpha_{i j m}}{2 l_{i j}^{2}}\left(w_{i}-w_{m}\right)+\frac{\cot \alpha_{m i j}}{2 l_{i j}^{2}}\left(w_{j}-w_{m}\right) .
\end{aligned}
$$

The definition of the discrete exterior derivative, on the other hand, is unchanged by the weights as it is a combinatorial operator, and thus only depends on the connectivity of the mesh [Munkres 1984].

\subsection{Discrete Laplace-Beltrami operator}

A particularly common operator, used in countless geometry processing tools, is the Laplace-Beltrami operator. Using the DEC framework, we can define it for weighted triangulations as the matrix $\Delta^{w}=d_{0}^{t} \star_{1} d_{0}$, where $d_{0}$ is the discrete exterior derivative on zero-forms [Desbrun et al. 2007]. This extends the familiar cotan-Laplacian matrix $\Delta^{\diamond}$ [Pinkall and Polthier 1993], which corresponds to the Laplace-Beltrami operator with zero weights (or, equivalently, constant weights). Note that $\Delta^{w}$ is a symmetric matrix providing the "weak" (i.e., integrated) version of the operator, while the "strong" (i.e., pointwise) version is simply the generally non-symmetric matrix $\star_{0}^{-1} \Delta^{w}$.

It bears pointing out that, due to the inherent primal-dual orthogonality of weighted triangulations, the operator $\Delta^{w}$ shares structural properties with the smooth Laplace-Beltrami operator such as symmetry, locality, scale invariance, as well as linear precision on flat domains [Wardetzky et al. 2007]. Based on our definition of dual metric, we can analyze further properties of this operator. Under the assumption that a sequence of triangle meshes converges to a smooth surface in the Hausdorff distance [Hildebrandt et al. 2006], the validity of dual metrics (Eq. (8)) implies that the weights become constant in the limit. As a consequence, $\Delta^{w}$ tends to the same operator in the limit as $\Delta^{\diamond}$, and thus converges to the smooth Laplace-Beltrami operator. We also notice that Eq. (8) corresponds to a sufficient condition introduced in [Glickenstein 2005] (Theorem 36) to enforce that the weighted Laplace-Beltrami operator is positive semi-definite with only constant functions in its kernel, and therefore satisfies Lax-Milgram theorem. We thus conclude that our definition of the Laplace-Beltrami operator for weighted triangulations shares the same properties as the cotan-Laplacian, while offering additional control over the construction of the dual diagram.

\subsection{Discussion}

While we showed how DEC can be conveniently leveraged to derive discrete differential operators on weighted triangulations, one can also use more classical approximation schemes. For instance, the weighted Laplace operator we introduced above was recently presented through a finite element derivation in [Desbrun et al. 2013] while a finite volume approach was used in [de Goes et al. 2013]. In these two related works, weights were approximating a divergence-free metric, i.e., a positive definite symmetric matrix field whose columns are divergence-free vector fields. Building upon this observation, the augmented metric can be interpreted as the piecewise Euclidian metric additively perturbed by a divergence-free matrix associated to the weights. This perturbation is visible in Eq. (13) as the extra term in $\star_{i j}$ added to $\star_{i j}^{\diamond}$.

Regarding numerical analysis, tight error bounds of DEC operators on weighted triangulations were previously addressed in [Mullen et al. 2011]. This work demonstrated that the inclusion of weights not only extends the typical finite element operators on meshes, but can also improve the accuracy of the discrete operators (without affecting the order of convergence). The authors proposed to compute weights as the minimizer of a family of energies that measures the discretization error of the diagonal Hodge star operators in Eqs. $(9,10,11)$. We finally point out that higher order accuracy can be achieved, if necessary, through the use of high-order approximations of the Hodge star [Buffa et al. 2010; Arnold 2013].

\section{METRIC CHARACTERIZATION}

While edge lengths and vertex weights are a convenient way to represent the metric of a weighted triangulation of a given connectivity, they are far from being the only set of geometrically relevant coefficients carrying the same information. In fact, depending on the targeted applications, more convenient descriptions of the weighted metric are available. In this section, we present three alternative representations of the metric which we will utilize in Sec. 6, each being a set of measurements on $T^{w}$ from which the lengths and weights of the weighted triangulation can be recovered (Fig. 3). We show their equivalence by describing an optimization over the set of augmented metrics $\mathbb{L}^{\mathbb{W}}$ to convert each of these metric representations back to the lengths-weights representation.

\subsection{Edge-Angle / Vertex-Area}

Our first metric characterization assumes that edge angle sums $\left\{\bar{\phi}_{i j}\right\}$ and positive dual cell areas $\left\{\bar{a}_{* i}\right\}$ of $T^{w}$ are given, along with its connectivity (Fig. 3, top). We show that one can recover the discrete metric $(l, w)$ of $T^{w}$ by solving two convex optimizations sequentially: first we recover the edge lengths $l$ from $\left\{\bar{\phi}_{i j}\right\}$, after which we deduce the weights $w$ based on $\left\{\bar{a}_{* i}\right\}$. 


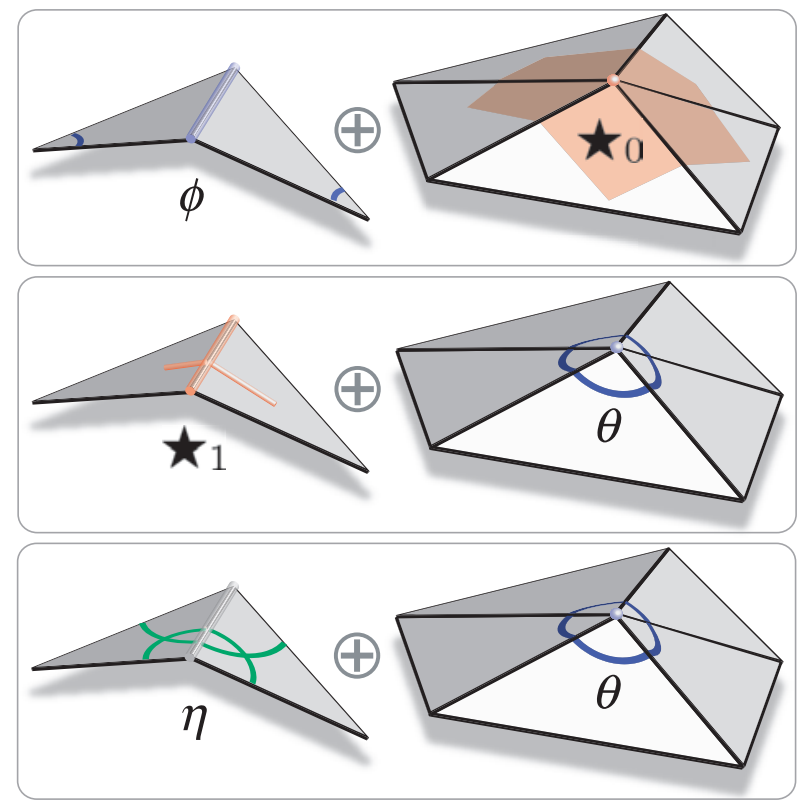

Fig. 3. Metric Characterizations of Weighted Triangulations: We offer three approaches to recover the augmented metric (edge lengths and vertex weights) of a weighted triangulation based on familiar geometric measurements: (top) angle sums $\phi$ per edge and discrete Hodge star values $\star_{0}$ per vertex corresponding to dual areas; (center) angle sums $\theta$ per vertex and discrete Hodge star values $\star_{1}$ per edge corresponding to primal-dual length ratios; (bottom) angle sums $\theta$ per vertex and inversive distances $\boldsymbol{\eta}$ per edge corresponding to the cosine of intersecting angles of vertex-centered circles.

The prescribed values $\left\{\bar{\phi}_{i j}\right\}$ and $\left\{\bar{a}_{* i}\right\}$ must satisfy necessary conditions in order to be associated to a discrete metric $(l, w)$. We first notice that $\bar{\phi}_{i j}$ is a sum of two triangle angles, which implies that $0<\bar{\phi}_{i j}<2 \pi$. Also, since our discrete surface is made out of Euclidean triangles for which interior angles sum to $\pi$, one has $\sum_{i j} \bar{\phi}_{i j}=\pi|F|$. Accounting for this linear constraint, the set of edge angles and vertex areas provide $|E|+|V|-1$ values, like $\mathbb{L}^{\mathbb{W}}$.

Circle Pattern Energy. Finding edge lengths $\left\{l_{i j}\right\}$ associated to the prescribed edge angle sums $\left\{\bar{\phi}_{i j}\right\}$ in $T^{w}$ is akin to the circle pattern problem [Rivin 1994; Bobenko and Springborn 2003]. We can thus solve for lengths by minimizing a convex energy $\mathcal{P}$ as introduced in [Bobenko et al. 2010]. We start by adopting the change of variables $u_{i j}=\log l_{i j}$. Note, however, that this logarithmic map transforms the triangle inequalities from linear functions of $\left\{l_{i j}\right\}$ (Eq. (7)) into non-linear functions of length scalings $\left\{u_{i j}\right\}$, and thus the convex set $\mathbb{L}$ becomes a non-convex set $\log \mathbb{L}$. We overcome this issue by extending $\log \mathbb{L}$ to a convex set as proposed in [Springborn et al. 2008], at the cost of violating the validity of primal metrics (Sec. 3.1). Within this space of variables $u=\left\{u_{i j}\right\} \in \log \mathbb{L}$, we define $\mathcal{P}$ as follows.

DEFINITION 10. The circle pattern energy $\mathcal{P}$ is defined as

$$
\begin{aligned}
\mathcal{P}(u) & =\sum_{i j k}\left(\Pi\left(\alpha_{i j k}\right)+\Pi\left(\alpha_{j k i}\right)+\pi\left(\alpha_{k i j}\right)\right) \\
& +\sum_{i j}\left(u_{i j}\left(\phi_{i j}-\bar{\phi}_{i j}\right)\right),
\end{aligned}
$$

where $Л(\cdot)$ denotes the Lobachevsky function [Milnor 1982], and the angles $\left\{\alpha_{i j k}\right\}$ and $\left\{\phi_{i j}\right\}$ depend on $u$.
We can now deduce the first and second derivatives of $\mathcal{P}$ as shown in the Appendix, and present the following theorem.

THEOREM 11. Assume that a connectivity and angle sums $\left\{\bar{\phi}_{i j}\right\}$ are given for $T^{w}$. Also assume that $0<\bar{\phi}_{i j}<2 \pi$ and $\sum_{i j} \bar{\phi}_{i j}=\pi|F|$. There exists at most one primal metric $\left\{l_{i j}\right\}$ (up to a global scaling) that recovers the prescribed edge angle sums.

ProOF. First, we notice that the gradient of $\mathcal{P}$ simplifies to

$$
\partial_{u_{i j}} \mathcal{P}=\phi_{i j}-\bar{\phi}_{i j}
$$

so that $\nabla_{u} \mathcal{P}=0$ iff the prescribed angles are recovered. We then point out that the Hessian of $\mathcal{P}$ is a positive semi-definite matrix with only constant functions in its null-space. This indicates that the energy $\mathcal{P}$ is convex in $u$. Since the extended domain of $\mathcal{P}$ is defined as a convex set, we conclude that the minimization of $\mathcal{P}$ is a convex optimization and, if a solution $\left\{l_{i j}=e^{u_{i j}}\right\}$ exists, it is unique up to a global scaling.

We can further determine the global scaling of $\left\{l_{i j}\right\}$ from the prescribed dual cell areas $\left\{\bar{a}_{* i}\right\}$, since their sum is equal to the total area of the mesh. We thus pick the properly scaled $\left\{l_{i j}\right\}$ so that:

$$
\sum_{i j k} a_{i j k}=\sum_{i} \bar{a}_{* i}
$$

Capacity Constrained Energy. Once the edge lengths are recovered, we compute the weights $w=\left\{w_{i}\right\}$ from the prescribed dual cell areas $\left\{\bar{a}_{* i}\right\}$ by observing that these values simply impose a "capacity" constraint for each vertex of $T^{w}$. Such a capacity constrained problem turns out to be a known optimal transport problem for which a variational principle was introduced in [Aurenhammer et al. 1998], and a closed form expression of the energy's Hessian was recently given in [de Goes et al. 2012]. Note that our context is different from [Aurenhammer et al. 1998; de Goes et al. 2012] since both of them were restricted to weighted Delaunay triangulations. Yet, we can modify their formulation to weighted triangulations with metrics in $\mathbb{L}^{\mathbb{W}}$ by introducing the energy $\mathcal{C}$.

DEFINITION 12. The capacity constraint energy $\mathcal{C}$ is defined as:

$$
\mathcal{C}(w)=\sum_{i} w_{i}\left(a_{* i}-\bar{a}_{* i}\right)-\sum_{i j k \in F}\left(\frac{d_{i j}^{3} h_{j k i}}{4}+\frac{d_{i j} h_{j k i}^{3}}{12}\right) .
$$

Note that the domain of $\mathcal{C}$ is the set of dual metrics $w=\left\{w_{i}\right\}$ for a given primal metric $\left\{l_{i j}\right\}$ in $\mathbb{L}$, and it is thus a convex polytope (Eq. (8)). We also point out that the second term of $\mathcal{C}$ is a weighted version of the Centroidal Voronoi Tessellation energy [Du et al. 1999] introduced in [Mullen et al. 2011]. With the derivatives of $\mathcal{C}$ given in the Appendix, we find weights $\left\{w_{i}\right\}$ satisfying the prescribed dual cell areas $\left\{\bar{a}_{* i}\right\}$ based on the following theorem.

THEOREM 13. Assume that a primal metric $\left\{l_{i j}\right\}$ and positive dual cell areas $\left\{\bar{a}_{* i}\right\}$ are given for $T^{w}$ so that Eq. (15) holds. There exists at most one dual metric $w=\left\{w_{i}\right\}$ (up to an additive constant) that recovers the prescribed dual cell areas.

PROOF. The gradient of $\mathcal{C}$ is found to be

$$
\partial_{w_{i}} \mathcal{C}=a_{* i}-\bar{a}_{* i},
$$

showing that $\nabla_{w} \mathcal{C}=0$ iff the prescribed dual cell areas are matched. The Hessian of $\mathcal{C}$ is one half of the Laplace-Beltrami operator $\Delta^{w}$ introduced in Sec. 2. This matrix is thus positive semidefinite for any metric in $\mathbb{L}^{\mathbb{W}}$, and its null-space only contains constant vectors, corresponding to the fact that the weights are determined up to an additive constant. Since the domain of $\mathcal{C}$ is convex, 
we finally conclude that the minimization of $\mathcal{C}$ is a convex optimization, and its solution is unique.

\subsection{Edge-Star / Vertex-Angle}

Our second metric representation is complimentary to the first one, as we now assume a primal-dual length ratio $\bar{\star}_{i j}$ given per edge, and an angle sum $\bar{\theta}_{i}$ given per vertex (Fig. 3, center). However, we will use a single variational principle to recover, up to scale, the discrete metric $(l, w)$ of a weighted triangulation $T^{w}$ that the length ratios and angle sums derive from.

Note that prescribing the sum of tip angles $\bar{\theta}_{i}$ around vertex $i$ is equivalent to constraining the discrete Gaussian curvature (or excess angle) $\kappa_{i}$ of the surface since $\kappa_{i}=2 \pi-\theta_{i}$ for non-boundary vertices. Moreover, since the tip angles of each triangle sum to $\pi$, a necessary condition for the existence of a metric $(l, w)$ for a given connectivity is that $\sum_{i} \bar{\theta}_{i}=\pi|F|$. On the other hand, prescribing the primal-dual length ratio $\nwarrow_{i j}$ is equivalent to specifying the diagonal Hodge-star for one-forms and consequently determines the discrete Laplace-Beltrami operator $\Delta^{w}$. Although the edge ratios and vertex angles involve $|E|+|V|-1$ values in total, they are invariant to scaling and thus this characterization only recovers the metric up to a global scaling.

Conformal Energy. Our formulation is based on the conformal energy, a functional that commonly arises in geometry processing [Pinkall and Polthier 1993; Mercat 2001; Desbrun et al. 2002; Lévy et al. 2002].

DEFINITION 14. The conformal energy of a piecewise-linear map $\mathbf{f}$ between a triangle mesh $\bar{T}$ and another mesh $T$ of identical connectivity is defined as:

$$
\mathcal{E}_{c}(\mathbf{f})=\frac{1}{2} \sum_{i j} \bar{\star}_{i j}\left\|\mathbf{f}_{i}-\mathbf{f}_{j}\right\|^{2}-\operatorname{Area}(\mathbf{f}),
$$

where the first term is the Dirichlet energy of $\mathbf{f}$ (measuring the smoothness of $\mathbf{f}$ in the metric of $\bar{T})$, and the second term is the area of the image of $\mathbf{f}$ (i.e., the total area of $T$ ).

Given that $\left\|\mathbf{f}_{i}-\mathbf{f}_{j}\right\|^{2}=l_{i j}^{2}$ where $\left\{l_{i j}\right\}$ are the edge lengths of $T$ and Area $(\mathbf{f})=\frac{1}{2}\left(\sum_{i j} \star_{i j} l_{i j}^{2}\right)=\frac{1}{2}\left(\sum_{i j} \star_{i j}^{\diamond} l_{i j}^{2}\right)$ as the total surface area is equal to the sum of all disjoint partition areas, no matter how the surface is partitioned, we can rewrite $\mathcal{E}_{c}$ as:

$$
\mathcal{E}_{c}\left(l^{2}\right)=\frac{1}{2} \sum_{i j}\left(\bar{\star}_{i j}-\star_{i j}\right) l_{i j}^{2}=\frac{1}{2} \sum_{i j}\left(\bar{\star}_{i j}-\star_{i j}\right) l_{i j}^{2} .
$$

Observe that the domain of $\mathcal{E}_{c}$ is the space $\mathbb{L}^{2}$ of squared edge lengths satisfying Eq. (7), and thus corresponds to a convex set homeomorphic to $\mathbb{L}$ [Zeng et al. 2012]. The partial derivatives of $\mathcal{E}_{c}$ are surprisingly simple despite the non-linear dependence of $\star_{i j}^{\diamond}$ on lengths (see Appendix):

$$
\partial_{l_{i j}^{2}} \mathcal{E}_{c}=\frac{1}{2}\left(\bar{\star}_{i j}-\star_{i j}^{\diamond}\right) \text {. }
$$

We thus conclude that the energy $\mathcal{E}_{c}$ is, in fact, a closed-form expression of the variational formulation introduced in [Zeng et al. 2012]: their energy, given in implicit form, admits the same gradient with respect to squared lengths. We can consequently reuse their results and present the following theorem.

THEOREM 15. Assume that the connectivity and length ratios $\left\{\nwarrow_{i j}\right\}$ for an unweighted mesh $T$ are given. There exists at most one primal metric $\left\{l_{i j}\right\}$ (up to a global scaling) that recovers the prescribed length ratios.
Proof. From Eq. (19), we have that $\left\{\bar{\star}_{i j}\right\}$ are recovered iff $\nabla_{l^{2}} \mathcal{E}_{c}=0$. Zeng et al. [2012] showed that the derivatives corresponding to the Hessian of $\mathcal{E}_{c}$ define a positive semi-definite matrix, with global scaling in its kernel, and thus $\mathcal{E}_{c}$ is a convex energy. Since the domain of $\mathcal{E}_{c}$ is a convex set, we conclude that the minimization of $\mathcal{E}_{c}$ in $l^{2}$ is a convex optimization and thus the solution $\left\{l_{i j}^{2}\right\}$ is unique up to a global scaling.

Notice that $\mathcal{E}_{c}$ is zero at its minimum since $\nabla_{l^{2}} \mathcal{E}_{c}=0$ implies the equality of (unweighted) stars, and hence a null conformal energy. However, in our context of weighted triangulations, we want to match the weighted Hodge star instead.

Curvature Constrained Conformal Energy. In order to extend the energy $\mathcal{E}_{c}$ to the augmented metric $(l, w)$, we consider a modified energy $\mathcal{L}$ that adds target vertex angles $\left\{\bar{\theta}_{i}\right\}$.

DEFINITION 16. The curvature constrained conformal energy $\mathcal{L}$ is defined as

$$
\mathcal{L}\left(l^{2}, w\right)=\mathcal{E}_{c}\left(l^{2}\right)-\frac{1}{2} \sum_{i} w_{i}\left(\bar{\theta}_{i}-\theta_{i}\right) .
$$

We provide an intuitive interpretation of our energy: $\mathcal{L}$ corresponds to the Lagrangian function of the minimization of $\mathcal{E}_{c}$ subject to constraints $\left\{\bar{\theta}_{i}=\theta_{i}\right\}$, where the weights play the role of (twice the) Lagrangian multipliers. Remarkably, the gradient of $\sum_{i} w_{i}\left(\bar{\theta}_{i}-\theta_{i}\right)$ with respect to $l^{2}$ contains exactly the weighted terms of the Hodge star for one-forms, and hence summing this with Eq. (19) turns the unweighted star $\star_{i j}^{\diamond}$ into a full Hodge star $\star_{i j}$.

THEOREM 17. The stationary points of $\mathcal{L}$ correspond to discrete metrics $(l, w)$ of $T^{w}$ that recover the prescribed measures $\left\{\bar{\theta}_{i}\right\}$ and $\left\{\bar{\star}_{i j}\right\}$.

PROOF. As detailed in the Appendix, the first derivatives of $\mathcal{L}$ are:

$$
\left\{\begin{array}{l}
\partial_{l_{i j}^{2}} \mathcal{L}=\frac{1}{2}\left(\bar{\star}_{i j}-\star_{i j}\right) \\
\partial_{w_{i}} \mathcal{L}=\frac{1}{2}\left(\theta_{i}-\bar{\theta}_{i}\right) .
\end{array}\right.
$$

Therefore, the prescribed edge ratios and vertex angles are recovered iff $\nabla_{l^{2}, w} \mathcal{L}=0$.

Note that our theorem does not address the uniqueness of the critical point of $\mathcal{L}$. In fact, the Hessian of $\mathcal{L}$ is in general indefinite, and $\mathcal{L}$ is thus non-convex. While the uniqueness of a solution of $\mathcal{L}$ remains an open question, we present instead additional analysis of its solution space.

COROLLARY 18. If a critical point of $\mathcal{L}$ exists with primal metric $\left\{l_{i j}\right\}$, then the dual metric is unique up to an additive constant.

Proof. We deduce from Eq. (21) that:

$$
\nabla_{l^{2}} \mathcal{L}=0 \Longrightarrow \star_{i j} l_{i j}^{2}=\bar{\star}_{i j} l_{i j}^{2} \forall i j \Longrightarrow \sum_{j \in \Omega_{i}} \star_{i j} l_{i j}^{2}=\sum_{j \in \Omega_{i}} \bar{\star}_{i j} l_{i j}^{2} \forall i \text {. }
$$

Since $\star_{i j}$ is linear in weights (Eq. (13)), we can express the deduced equalities as a Poisson linear system:

$$
\Delta^{\diamond} w=b,
$$

where $b_{i}=\sum_{j \in \Omega_{i}} l_{i j}^{2}\left(\bar{\star}_{i j}-\star_{i j}^{\diamond}\right)$. Note that the kernel of $\Delta^{\diamond}$ only contains constant vectors. Thus, for a critical point of $\mathcal{L}, b$ is in the range of $\Delta^{\diamond}$, and there is a unique set of weights (up to an additive constant) satisfying Eq. (22). 
Finally, we show that the space of weighted Hodge stars for oneforms is strictly larger than the space of unweighted Hodge stars, confirming the flexibility added by weighted triangulations in the discretization of differential operators.

COROllary 19. Assume a weighted mesh $\widehat{T}^{w}$ of metric $(\widehat{l}, \widehat{w})$ is given, with non-constant weights. Then, for any unweighted mesh $T$ with the same connectivity as $\widehat{T}^{w}: \star_{i j} \neq \widehat{\star}_{i j} \forall i j$.

PROOF. Our proof is by contradiction. Suppose there exists a triangle mesh $T$ of edge length $l=\left\{l_{i j}\right\}$ and constant weights $w=\left\{w_{i}\right\}$ such that the equality $\star_{i j}=\widehat{\star}_{i j}$ holds. The length assignment $l$ is then a global minimum of the convex energy $\mathcal{E}_{c}$ with prescribed discrete Hodge star $\bar{\star}_{i j} \equiv \widehat{\star}_{i j}$. Note, however, that $\widehat{l}=\left\{\widehat{l}_{i j}\right\}$ is also a global minimizer of $\mathcal{E}_{c}$ since $\mathcal{E}_{c}(\widehat{l})=\mathcal{E}_{c}(l)=0$ (see Eq. (18)). Due to the convexity of $\mathcal{E}_{c}$, we conclude that $\widehat{l}=s l$, where the scalar $s$ indicates a global scaling factor. Now, observe that the linear system in Eq. (22) for these two meshes differs just in $\widehat{b}=s^{2} b$. Thus $\widehat{w}=s^{2} w$ and is constant, contradicting the initial assumption that $\widehat{w}$ is non-constant.

\subsection{Inversive Distance Circle Packing}

Our third and last metric characterization involves the concept of circle packing, a topic that has attracted a lot of efforts since Thurston [1976] linked it to conformal mapping (see [Stephenson 2003] for a survey). We show that the discrete metric $(l, w)$ of $T^{w}$ can be determined by solving an inversive distance circle packing problem, from prescribed angle sums $\left\{\bar{\theta}_{i}\right\}$ per vertex, and additional positive values $\left\{\eta_{i j}\right\}$ per edge known as the inversive distances (Fig. 3, bottom).

Overview. One can think of the weight $w_{i}$ as the square of the radius of an intrinsic circle centered at vertex $i$. These circles define, within each triangle's supporting plane, a unique circle orthogonal to them, and the center of this circle per triangle is our dual vertex $\mathbf{c}_{i j k}$ as shown, e.g., in [Glickenstein 2005]. It is therefore no surprise that weighted triangulations are relevant in the context of circle packing. The circle packing problem defines a relation between edge lengths and vertex weights of the form:

$$
l_{i j}^{2}=w_{i}+w_{j}+2 \sqrt{w_{i} w_{j}} \eta_{i j} .
$$

This resembles the law of cosines where $\eta_{i j}$ indicates how neighboring circles intersect. By scaling vertex circles, one can then deform edge lengths while keeping the values $\left\{\eta_{i j}\right\}$ invariant. The original circle packing problem (used, e.g., in [Jin et al. 2008]) assumes positive weights and intersecting circles with acute angles (i.e., $0 \leq \eta \leq 1$ ), while inversive distance circle packing [Yang et al. 2009] extends this concept to non-intersecting circles (i.e., $\eta \geq 0$ ). Arbitrarily signed weights can further be treated as discussed in [Aurenhammer 1987; Pedoe 1988].

Circle Packing Energy. Recent results [Guo 2009; Luo 2010] have shown the existence of an energy (with no known explicit form) that recovers, at its critical point, an augmented metric $(l, w)$ from vertex angles $\left\{\bar{\theta}_{i}\right\}$ and inversive distances $\left\{\eta_{i j}\right\}$. They also showed that this energy relies on the change of variables $u_{i}=$ $\frac{1}{2} \log w_{i}$, and Eq.(23) is then rewritten as:

$$
l_{i j}^{2}=e^{2 u_{i}}+e^{2 u_{j}}+2 e^{u_{i}+u_{j}} \eta_{i j} .
$$

Yang et al. [2009] later derived that the Hessian of this energy as a function of vertex scalings $\left\{u_{i}\right\}$ is the weighted Laplacian $\Delta^{w}$ defined in Sec. 4. We can then use the augmented metric set $\mathbb{L}^{\mathbb{W}}$ in order to guarantee that the operator $\Delta^{w}$ is positive semi-definite and thence that the circle packing energy is convex. Lastly, Guo [2009] proved that the space of vertex scalings is convex once $\eta \geq 0$, and the minimization of the circle packing energy is thus reduced to a convex optimization. In the following theorem, we summarize the metric characterization of $T^{w}$ based on the inversive distance circle packing.

THEOREM 20. Assume that connectivity, vertex angles $\left\{\bar{\theta}_{i}\right\}$ and positive inversive distances $\left\{\eta_{i j}\right\}$ are given for $T^{w}$. There exists a convex energy such that its unique minimum (if any) is reached at vertex scalings $\left\{u_{i}\right\}$ defining the augmented metric $(l, w)$ that recovers the prescribed values $\left\{\bar{\theta}_{i}\right\}$ and $\left\{\eta_{i j}\right\}$.

\subsection{Discussion}

Our metric characterizations relate to and tie together several problems and energies that commonly arise in geometry processing. Circle patterns (Eq. (14)) and conformal energies (Eq. (18)), for instance, are standard methods to compute mesh parameterizations [Lévy et al. 2002; Kharevych et al. 2006]. We employed the little known edge-scaling-based version of the circle pattern energy from [Bobenko et al. 2010] instead of the original circumradiusbased energy [Bobenko and Springborn 2003]. In fact, the relevance of the energies $\mathcal{P}$ (Eq. (14)) and $\mathcal{E}_{c}$ (Eq. (18)) are not a mere coincidence. Recently, Luo [Luo 2010] showed the existence of a whole family of implicit energies that uniquely characterize the edge lengths of a triangle mesh, for which $\mathcal{P}$ and $\mathcal{E}_{c}$ correspond to two special cases (in Luo's notation, $h=0$ and $h=-2$, respectively). Our work revealed simple closed-form expressions for these energies. Moreover, we made use of recent work on the capacity constrained problem, another example of a core theoretical result with applications in graphics such as displacement interpolation [Mérigot 2011] and blue noise generation [de Goes et al. 2012]. While previous work addressed capacity constraints on weighted Delaunay triangulations (for which connectivity is induced by the weights), we instead extend this formulation to any weighted triangulations with metric $(l, w) \in \mathbb{L}^{\mathbb{W}}$.

The representation of weighted triangulations as circle packings also offers an interesting counterpart to the discrete conformal equivalence of unweighted triangle meshes introduced in [Springborn et al. 2008]: while they employ a primal mesh with $|E|$ values to define the metric, $|E|-(|V|-1)$ cross ratios to define the conformal invariants, and $|V|-1$ curvatures to complete the picture, we assume a weighted triangulation instead, with $|E|+|V|-1$ values defining the metric, $|E|$ inversive distances defining the conformal invariants, and $|V|-1$ curvatures to complete the picture. These two approaches are, however, closely related and come from special cases of a more general notion of discrete conformal equivalence introduced in [Glickenstein 2011]. We can in fact derive an additional insight connecting these two conformal structures. Consider the family of meshes $T^{w}$ with primal metric $\left\{\widehat{l}_{i j}\right\}$ and constant dual metric $\left\{\widehat{w}_{i} \equiv \gamma\right\}$. In this particular case, inversive distances can be rewritten as $\eta_{i j}=\frac{1}{2 \gamma} \widehat{l}_{i j}^{2}-1$, and plugging this form into Eq. (24) we have:

$$
\begin{aligned}
l_{i j}^{2} & =e^{2 u_{i}}+e^{2 u_{j}}+2 e^{u_{i}+u_{j}}\left(\frac{1}{2 \gamma} \widehat{l}_{i j}^{2}-1\right) \\
& =\left(e^{u_{i}}-e^{u_{j}}\right)^{2}+\frac{1}{\gamma} e^{u_{i}+u_{j}} \widehat{l}_{i j}^{2} .
\end{aligned}
$$

Note that the second term of this expression corresponds to a mapping that preserves length cross ratio as introduced in [Springborn et al. 2008], while the complete expression keeps the inversive distance invariant. Therefore, inversive distance circle packing can be interpreted as a generalization of discrete conformal equivalence 
for weighted triangulations that maintains a packing structure on the mesh, while trading off preservation of length cross ratios and uniformity of vertex scalings.

\section{APPLICATIONS}

Weighted triangulations are amenable to most geometry processing tasks due to their added flexibility for the construction of discrete operators through the design of an extended set of orthogonal dual meshes. In this section, we leverage the insights developed in our work to provide concrete examples of weighted triangulations applied to meshing. In particular, we show how to improve upon recent methods in mesh generation and architecture modeling.

\subsection{Self-Supporting Meshes}

Self-supporting meshes are architectural structures that support external vertical loads with no need for additional reinforcement. The design and analysis of such structures has received attention in graphics in recent years [Vouga et al. 2012; Panozzo et al. 2013; Liu et al. 2013; de Goes et al. 2013]. In particular, both the work of [Liu et al. 2013] and [de Goes et al. 2013] noticed that any simply-connected self-supporting mesh $T^{w}$ described as a piecewise linear height function $\left\{z_{i}\left(\mathbf{x}_{i}\right)\right\}$ withstands an external load $\left\{\rho_{i}\right\}$ iff its orthographically projected planar mesh accepts a dual metric $w=\left\{w_{i}\right\}$ with positive dual lengths $\left(l_{i j}^{*} \geq 0\right)$ and satisfying the equilibrium equation:

$$
\Delta^{w} z=\rho .
$$

Moreover, Vouga et al. [2012] showed that the ratio of a vertex load $\rho_{i}$ and its dual cell area $a_{* i}$ induced by the dual metric $w$ is equivalent to the concept of relative mean curvature borrowed from the theory of isotropic geometry [Pottmann et al. 2009]. Based on this observation and on Eq. (5), Liu et al. [2013] proposed to generate smooth self-supporting meshes through an iterative process that estimates vertex weights by approximating $a_{* i}=\rho_{i} / H$ for every vertex $i$, where $H$ indicates a constant relative mean curvature.

We point out that the construction of a dual metric $w$ from a given load function $\rho$ and constant relative mean curvature $H$ is akin to the capacity-constrained problem described in Sec. 5.1. We can indeed set $\bar{a}_{* i} \equiv \rho_{i} / H$ and solve for the dual metric $w$ associated to a

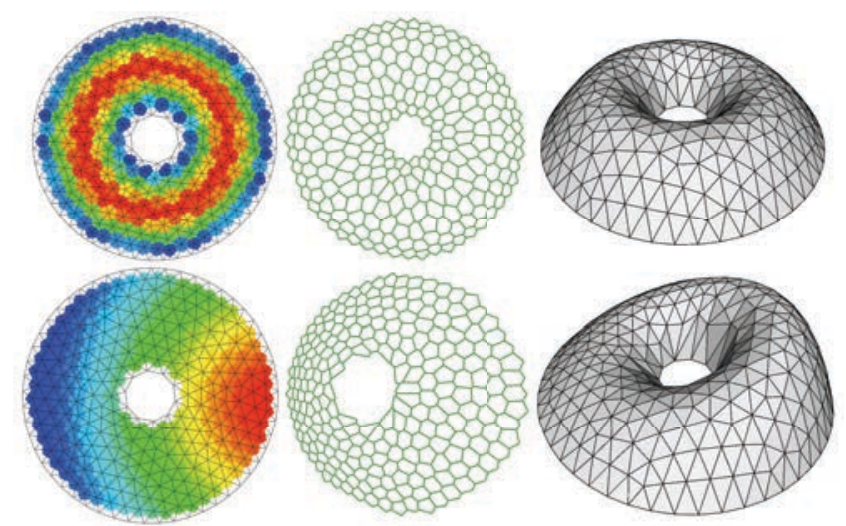

Fig. 4. Modelling self-supporting meshes. Based on the distribution of vertical loads on a planar mesh (left, color ramp indicates load intensity ranging from blue to red) and setting a target relative mean curvature ( $H=5$ for both examples), we construct self-supporting meshes by first recovering a dual metric $w$ that minimizes the energy $\mathcal{C}$ in Eq. (16) (center), and then recovering a height function from Eq. (25) (right).
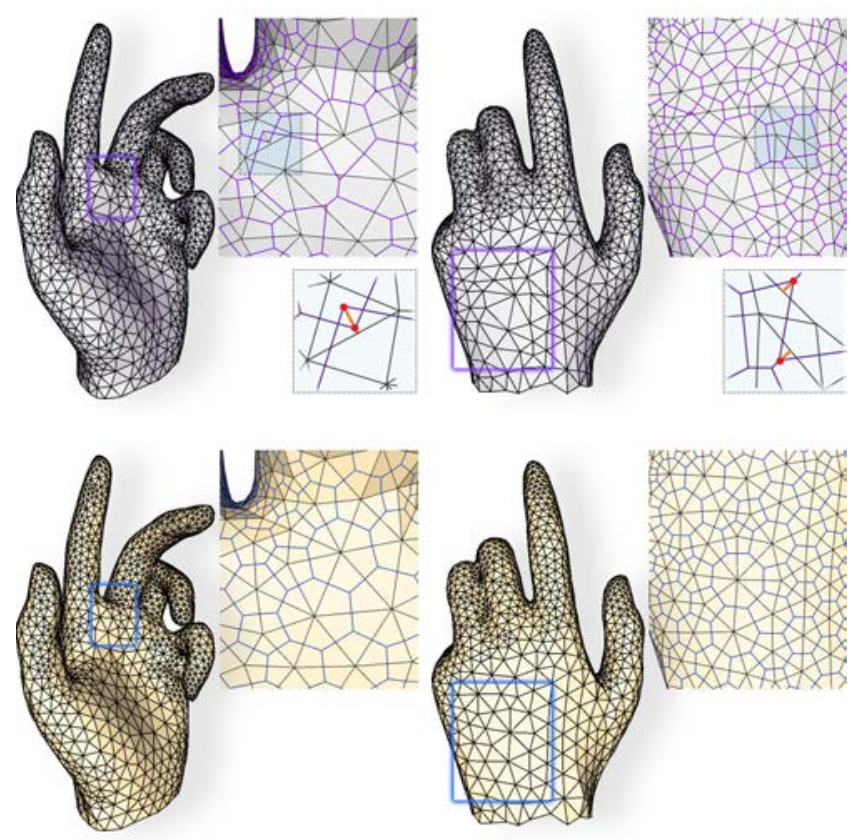

Fig. 5. Well-centered Meshes. The hand model contains many triangles with offcentered circumcenters. Even after optimizing the dual metric $w$ based on Eq. (27a), most dual vertices get closer to their respective triangle barycenter (top), but a few still remain outside their triangles (see red lines). We can generate a well-centered mesh by optimizing the same energy for both vertex positions and weights, while retaining the original surface shape (bottom).

planar primal mesh by minimizing the energy $\mathcal{C}$ (Eq. (16)) through an efficient Newton's solver [Nocedal and Wright 1999]. We can further enforce positive dual lengths either by adding them as inequality constraints (as in [de Goes et al. 2013]) or by performing edge flips (as in [Liu et al. 2013]). Once the 2D primal-dual mesh is found, we can finally construct a self-supporting height function by solving Eq. (25). As illustrated in Fig. 4, our approach offers a novel tool for modeling self-supporting meshes based on prescribed distribution of vertex loads. Our implementation is based on the Ipopt library [Wächter and Biegler 2006] (with default parameters) and took three iterations to convergence.

\subsection{Well-centered Meshes}

In various meshing contexts, it is desirable to place dual vertices inside their associated triangle while retaining orthogonality to the primal mesh [Elcott et al. 2007; Batty et al. 2010]. However, generating such "well-centered" meshes with circumcentric duals are challenging [VanderZee et al. 2010]. Alternatively, Mullen et al. [2011] proposed the use of weighted circumcenters to construct well-centered triangulations in the plane. Their approach is based on the minimization of the functional $\mathcal{M}$ that evaluates the cost of coalescing mesh triangles $i j k$ into their respective dual points $\mathbf{c}_{i j k}$ :

$$
\mathcal{M}(\mathbf{x}, w)=\sum_{i j k \in F} \int_{i j k}\left\|\mathbf{y}-\mathbf{c}_{i j k}\right\|^{2} d \mathbf{y} .
$$

In the case of surface meshes, the authors restricted the minimization of $\mathcal{M}$ only with respect to weights, and showed that the weighted circumcenters move towards their respective triangle barycenter as expected. 
In order to generate well-centered meshes over non-flat domains, we first point out that the energy $\mathcal{M}$ can be expressed as a sum of two terms:

$$
\left\{\begin{array}{l}
\mathcal{S}_{\mathbf{b}}(\mathbf{x}, w)=\sum_{i j k} a_{i j k}\left\|\mathbf{b}_{i j k}-\mathbf{c}_{i j k}\right\|^{2} \\
\mathcal{V}(\mathbf{x})=\sum_{i j k} \int_{i j k}\left\|\mathbf{y}-\mathbf{b}_{i j k}\right\|^{2} d \mathbf{y}
\end{array}\right.
$$

Note that the first energy $\mathcal{S}_{\mathbf{b}}$ measures how close the dual metric $w$ places the dual vertices $\left\{\mathbf{c}_{i j k}\right\}$ with respect to triangle barycenters $\left\{\mathbf{b}_{i j k}\right\}$, capturing the key property promoted by [Mullen et al. 2011]. The second energy $\mathcal{V}$, on the other hand, corresponds to the sum of second moments of the mesh triangles, and favors the formation of evenly sized triangles. While Mullen et al. [2011] recommend the sum of these energies in the context of Hodge star optimization, we propose to use only $\mathcal{S}_{\mathbf{b}}$ to construct well-centered surface meshes. We minimize the energy $\mathcal{S}_{\mathbf{b}}$ by alternating optimization of mesh positions $\left\{\mathbf{x}_{i}\right\}$ and weights $\left\{w_{i}\right\}$. We use a LBFGS method [Nocedal and Wright 1999] to optimize vertex positions. For weight optimization, we exploit our construction of weighted orthogonal duals as displacements of the circumcentric dual (Eq. (1)), and point out that this minimization amounts precisely to finding a gradient field $\nabla w$ on $T$ that best matches (in the $L_{2}$ sense) a given vector per triangle. This particular problem is well studied (e.g., in [Tong et al. 2003]) and can be efficiently solved with a single linear system:

$$
\Delta^{\diamond} w=\nabla \cdot\left(\mathbf{c}^{\diamond}-\mathbf{b}\right),
$$

where $\left[\nabla \cdot\left(\mathbf{c}^{\diamond}-\mathbf{b}\right)\right]_{i}=\sum_{i j k \in \Omega_{i}}\left(\mathbf{c}_{i j k}^{\diamond}-\mathbf{b}_{i j k}\right)^{t} \mathbf{e}_{j k}^{\perp}$. Fig. 5 compares the results of weight-only versus position and weight optimization. Note that the weights alone still generate off-centered elements, while our full optimization places dual vertices at the barycenters, and also improves the aspect ratio of the triangles. In our experiments, we used our own implementation of the L-BFGS method with limited memory of size 5, and the library [Davis 2011] as our sparse linear solver. As the stopping criteria, we set a convergence tolerance of $10^{-10}$ which was achieved in 10-20 iterations.

\subsection{Incircle and Sphere Packing}

We discussed in Sec. 5.3 the inversive distance circle packing problem and its link to weighted triangulations. A singular case of circle packing occurs when vertex-centered circles are tangent, i.e., when $\eta=1$. This case, called sphere packing, has been used in surface modeling [Schiftner et al. 2009] to design torsion-free structures relevant for architecture. In this same work, the authors noted that

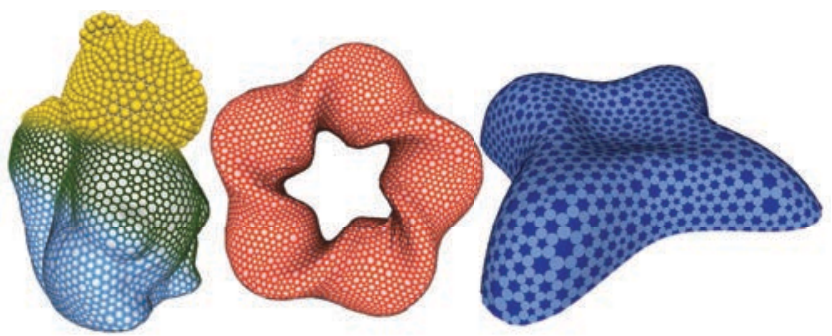

Fig. 6. Incircle and sphere packing on surfaces. Examples of incircle packing on triangulated surfaces with boundary, non-trivial topology, and curved shapes. Incircle packing directly induces sphere packing and an orthogonal dual structure (left). a sphere packing corresponds to a triangle mesh with pairwise tangent incircles, a property that induces an orthogonal dual structure where incenters are dual vertices. We can thus modify Eq. (27a) to generate incircle and sphere packing through minimization of the energy:

$$
\mathcal{S}_{\mathbf{i}}(\mathbf{x}, w)=\sum_{i j k} a_{i j k}\left\|\mathbf{i}_{i j k}-\mathbf{c}_{i j k}\right\|^{2},
$$

where $\mathbf{i}_{i j k}$ denotes the incenter of triangle $i j k$. One can then alternate the minimization of this energy with respect to weights $w_{i}$ using the Poisson solve in Eq. (28), and with respect to points $\mathbf{x}_{i}$ using a L-BFGS method [Nocedal and Wright 1999] (with the same parameters as in Sec. 6.2). This approach turns out to be much more robust than the two energies suggested in [Schiftner et al. 2009]: while theirs require an initial guess that is close to be an incircle packing in order to return valuable results, our approach is robust to local minima and handles bad initial guesses quite well as Fig. 7 demonstrates. Our approach successfully generates incircle packings on highly curved shapes, with or without boundaries, and with arbitrary topology as shown in Fig. 6.
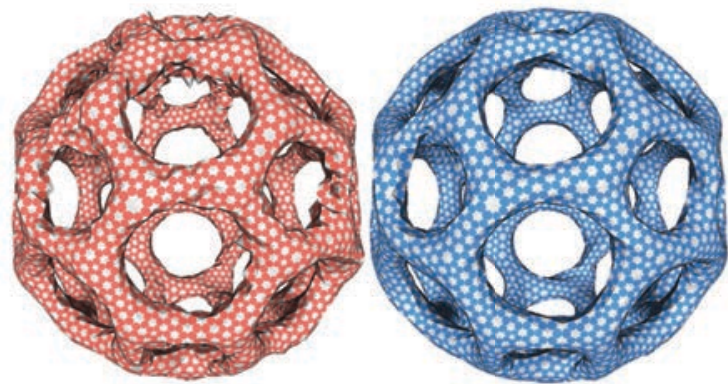

Fig. 7. Incircle Packing Comparison. While the optimization method of [Schiftner et al. 2009] (left) is sensitive to bad initial meshes, our approach (right) reliably generates incircle packing on surfaces of arbitrary shape and topology.

\section{CONCLUSION AND FUTURE WORK}

In this paper, we advocate the use of weighted triangulations in geometry processing: the additional weights on vertices provide flexibility in the design of basic tools used in our community. While our work focused on defining foundations for weighted triangulations and demonstrating a number of applications in the context of mesh design, we expect that additional benefits will be derived from further studies. On the theoretical side, we are now investigating necessary conditions for the existence of critical points of our metric characterization in Sec. 5. In particular, we conjecture that any weighted Hodge star for one-forms requires at most one set of Gaussian curvatures to be fully determined. This conjecture, if proven correct, would extend the global rigidity theorem presented in [Zeng et al. 2012] from triangle meshes to weighted triangulations. Discrete metrics of non-orthogonal primal-dual meshes and polyhedral meshes are also of interest, as well as the extension of our construction to non-intrinsic dual vertices. Further insights may be gathered by linking our characterizations to the use of weighted circle patterns [Springborn 2008; Dimitrov 2012]. Finally, on the practical side, we believe our geometric dual meshes can bring added robustness to numerous computational tools, and lead to new approaches for geometry processing as demonstrated in Sec. 6; in particular, it may help extend recent methods that process shapes 
not solely based on the usual vertex positions, but on discrete differential geometric quantities [Lipman et al. 2005; Crane et al. 2011; Wang et al. 2012].

\section{ACKNOWLEDGMENTS}

The authors wish to acknowledge support through a $\mathrm{PhD}$ Google Fellowship and a grant (CCF-1011944) from the National Science Foundation. Pensatore model (Fig. 1), hand model (Fig. 5), squirrel model (Fig. 6) are courtesy of Aim@ Shape; Lilium tower (Fig. 6) is courtesy of Evolute GmbH; and twisted torus (Fig.6 6) is courtesy of Keenan Crane. Thanks to SantiagoV. Lombeyda for his help with figures.

\section{REFERENCES}

Abraham, R., Marsden, J. E., And RatiU, T. 1988. Manifolds, tensor analysis, and applications, Second ed. Applied Mathematical Sciences, vol. 75. Springer-Verlag.

Alliez, P., Cohen-Steiner, D., Yvinec, M., And Desbrun, M. 2005. Variational tetrahedral meshing. ACM Trans. Graph. 24, 3 (July), 617-625.

ARNOLD, D. N. 2013. Spaces of finite element differential forms. In Analysis and Numerics of Partial Differential Equations, U. Gianazza, F. Brezzi, P. Colli Franzone, and G. Gilardi, Eds. Springer, 117-140.

Aurenhammer, F. 1987. Power diagrams: properties, algorithms and applications. SIAM J. Comput. 16, 1, 78-96.

Aurenhammer, F., Hoffmann, F., AND ARonov, B. 1998. Minkowski-type theorems and least-squares clustering. Algorithmica 20, 1, 61-76.

Batty, C., Xenos, S., And Houston, B. 2010. Tetrahedral embedded boundary methods for accurate \& flexible adaptive fluids. Comp. Graph. Forum (Eurographics) 29, 695-704.

Bobenko, A., Pinkall, U., And Springborn, B. 2010. Discrete conformal maps and ideal hyperbolic polyhedra. arXiv:1005.2698.

Bobenko, A. I. AND Springborn, B. A. 2003. Variational principles for circle patterns and Koebe's theorem. Trans. Amer. Math. Soc. 356, 659-689.

Bossavit, A. 1998. Computational Electromagnetism. Academic Press, Boston.

Buffa, A., SANGalli, G., AND VÁzQuez, R. 2010. Isogeometric analysis in electromagnetics: B-splines approximation. Computer Methods in Applied Mechanics and Engineering 199, 1720, 1143 - 1152.

Chow, B. AND LuO, F. 2003. Combinatorial Ricci flows on surfaces. $J$. Diff. Geom. 63, 1, 97-129.

COLIN DE VERDIÈRE, Y. 1991. Un principe variationnel pour les empilements de cercles. Inventiones Mathematicae 104, 655-669.

Crane, K., Pinkall, U., AND Schröder, P. 2011. Spin transformations of discrete surfaces. ACM Trans. Graph. (SIGGRAPH) 30, 104:1104:10.

DAVIS, T. A. 2011. Algorithm 915, SuiteSparseQR: Multifrontal multithreaded rank-revealing sparse QR factorization. ACM Trans. Math. Softw. 38, 8:1-8:22.

De Goes, F., Alliez, P., Owhadi, H., And Desbrun, M. 2013. On the equilibrium of simplicial masonry structures. ACM Trans. Graph. (SIGGRAPH) 32.

de Goes, F., Breeden, K., Ostromoukhov, V., and Desbrun, M. 2012. Blue noise through optimal transport. ACM Trans. Graph. (SIGGRAPH Asia) 31, 171:1-171:11.

Desbrun, M., Donaldson, R., And Owhadi, H. 2013. Modeling across scales: Discrete geometric structures in homogenization and inverse homogenization. In Multiscale analysis and nonlinear dynam- ics: from genes to the brain, M. Z. Pesenson, Ed. Reviews of Nonlinear Dynamics and Complexity, vol. 8. Wiley. Extended version of arXiv:0904.2601 [math.AP], 2009.

Desbrun, M., Kanso, E., And Tong, Y. 2007. Discrete differential forms for computational modeling. In Discrete Differential Geometry, A. Bobenko and P. Schröder, Eds. Springer.

Desbrun, M., Meyer, M., And Alliez, P. 2002. Intrinsic Parameterizations of Surface Meshes. Comp. Graph. Forum (Eurographics) 21.

Dimitrov, N. 2012. Positively weighted Delaunay triangulations and their circle patterns as critical points of the hyperbolic volume functional. unpublished.

Du, Q., Faber, V., And Gunzburger, M. 1999. Centroidal Voronoi tessellations: Applications and algorithms. SIAM Rev. 41, 637-676.

DYER, R. AND SCHAEFER, S. 2009. Circumcentric dual cells with negative area. Tech. Rep. TR2009-06, School of Computing Science, Simon Fraser University.

Elcott, S., Tong, Y., Kanso, E., Schröder, P., And Desbrun, M. 2007. Stable, circulation-preserving, simplicial fluids. ACM Trans. Graph. 26, 1 (Jan.).

Fisher, M., Springborn, B., Schröder, P., And Bobenko, A. I. 2007. An algorithm for the construction of intrinsic Delaunay triangulations with applications to digital geometry processing. Computing 81, 2-3 (Nov.), 199-213

GLICKEnSTEIn, D. 2005. Geometric triangulations \& discrete Laplacians on manifolds. arXiv.org:math/0508188.

GLickenstein, D. 2011. Discrete conformal variations and scalar curvature on piecewise flat two- and three-dimensional manifolds. J. Differential Geom. 87, 201-238.

Grady, L. J. And Polimeni, J. R. 2010. Discrete Calculus: Applied Analysis on Graphs. Springer.

GuO, R. 2009. Local rigidity of inversive distance circle packing. arXiv:0903.1401v2.

Hildebrandt, K., Polthier, K., And Wardetzky, M. 2006. On the convergence of metric and geometric properties of polyhedral surfaces. Geometria Dedicata 123, 89-112.

Hirani, A. N., Kalyanaraman, K., and VanderZee, E. B. 2013. Delaunay Hodge star. Computer-Aided Design 45, 2, 540-544.

Jin, M., KIM, J., LuO, F., AND GU, X. 2008. Discrete surface ricci flow. IEEE Tran. Vis. Comp. Graph. 14, 5 (sept.-oct.), 1030-1043.

Kharevych, L., Springborn, B., AND SchröDer, P. 2006. Discrete conformal mappings via circle patterns. ACM Trans. Graph. 25, 2, 412438.

Lévy, B., Petitjean, S., Ray, N., and Maillot, J. 2002. Least squares conformal maps for automatic texture atlas generation. ACM SIGGRAPH 21, 3, 362-371.

Lipman, Y., Sorkine, O., Levin, D., AND COHEN-OR, D. 2005. Linear rotation-invariant coordinates for meshes. ACM Trans. on Graph. (SIGGRAPH) 24, 3, 479-487.

Liu, Y., Hao, P., Snyder, J., Wang, W., And Guo, B. 2013. Computing self-supporting surfaces by regular triangulation. ACM Trans. Graph. (SIGGRAPH) 32.

LuO, F. 2010. Rigidity of polyhedral surfaces, III. arXiv:1010.3284v1.

MACNEAL, R. 1949. The solution of partial differential equations by means of electrical networks. Ph.D. thesis, Caltech.

Mercat, C. 2001. Discrete Riemann surfaces and the Ising model. Comm. Math. Phys. 218, 177-216.

MÉRIGOT, Q. 2011. A multiscale approach to optimal transport. Comput. Graph. Forum 30, 5, 1583-1592. 
Meyer, M., Desbrun, M., Schröder, P., ANd Barr, A. H. 2002. Discrete differential-geometry operators for triangulated 2-manifolds. In Proc. VisMath. 35-57.

MilnOR, J. 1982. Hyperbolic geometry: the first 150 years. Bul. Amer. Math. Soc. 6, 1, 9-24.

Mullen, P., Memari, P., De Goes, F., And Desbrun, M. 2011. Hot: Hodge-optimized triangulations. ACM Trans. Graph. (SIGGRAPH) 30, 103:1-103:12.

MunkRes, J. R. 1984. Elements of Algebraic Topology. Addison-Wesley.

Nocedal, J. AND Wright, S. J. 1999. Numerical optimization. Springer Verlag.

Panozzo, D., Block, P., And Sorkine-Hornung, O. 2013. Designing unreinforced masonry models. ACM Trans. Graph. 32, 4(July), 91:191:12.

Pedoe, D. 1988. Geometry, a comprehensive course, 2nd ed. Dover Publications.

Pinkall, U. AND Polthier, K. 1993. Computing discrete minimal surfaces and their conjugates. Experimental Mathematics 2, 15-36.

Polthier, K. AND PREUSS, E. 2003. Identifying vector field singularities using a discrete hodge decomposition. In Visualization and Mathematics III. Springer-Verlag, 113-134.

Pottmann, H., Grohs, P., And Mitra, N. 2009. Laguerre minimal surfaces, isotropic geometry and linear elasticity. Adv. Comp. Math, $391-419$.

REGGE, T. 1961. General relativity without coordinates. Nuovo Cim. 19, 3, $558-571$.

RIVIN, I. 1994. Euclidean structures on simplicial surfaces and hyperbolic volume. Annals of Math. 139, 3 .

Schiftner, A., Höbinger, M., Wallner, J., And Pottmann, H. 2009. Packing circles and spheres on surfaces. ACM Trans. Graph. 28, 5 (Dec.), 139:1-139:8.

SPRINGBORN, B. 2008. A variational principle for weighted Delaunay triangulations and hyperideal polyhedra. J. Differential Geom. 78, 2.

Springborn, B., Schröder, P., AND Pinkall, U. 2008. Conformal equivalence of triangle meshes. ACM Trans. Graph. (SIGGRAPH) 27, 77:1-77:11.

Stephenson, K. 2003. Circle packing: A mathematical tale. Notices Amer. Math. Soc. 50, 11, 1376-1388.

THURSTON, W. 1976. Geometry and topology of 3-manifolds.

Tong, Y., Lombeyda, S., Hirani, A. N., and Desbrun, M. 2003. Discrete multiscale vector field decomposition. ACM Trans. Graph. 22, 3 (July), 445-452.

VAnderZee, E., Hirani, A. N., Guoy, D., AND Ramos, E. 2010. Wellcentered triangulation. SIAM Journal on Scientific Computing 31, 6, 4497-4523.

Vouga, E., Höbinger, M., Wallner, J., And Pottmann, H. 2012. Design of self-supporting surfaces. ACM Trans. Graph. 31, 4, 87:187:11

WÄCHTER, A. AND BIEGLER, L. T. 2006. On the implementation of an interior-point filter line-search algorithm for large-scale nonlinear programming. Math. Program. 106, 1, 25-57.

WANG, Y., LIU, B., AND TONG, Y. 2012. Linear surface reconstruction from discrete fundamental forms on triangle meshes. Comp. Graph. Forum 31, 8, 2277-2287.

Wardetzky, M., Mathur, S., Kälberer, F., And Grinspun, E. 2007. Discrete Laplace operators: No free lunch. In Proc. of Symp. on Geo. Proc. 33-37.

YANG, Y.-L., GuO, R., LuO, F., Hu, S.-M., AND Gu, X. 2009. Generalized discrete Ricci flow. Comput. Graph. Forum 28, 7, 2005-2014.
Zeng, W., Guo, R., Luo, F., And Gu, X. 2012. Discrete heat kernel determines discrete Riemannian metric. Graphical Models 74, 4, 121129.

\section{Appendix: Derivatives}

In this section, we give explicit expressions for the derivatives of the energies introduced in Sec. 5. We spell out these expressions per triangle (we will use a triangle $i j k$ of circumradius $R$ ) to facilitate direct implementation. We will also use two identities derived from the cosine law:

$\partial_{l_{i j}^{2}} \alpha_{k i j}=-\frac{\cot \alpha_{i j k}}{2 l_{i j}^{2}}, \partial_{l_{i j}^{2}} \alpha_{j k i}=\frac{\cot \alpha_{i j k}+\cot \alpha_{k i j}}{2 l_{i j}^{2}}=\frac{1}{4 a_{i j k}}$.

Circle-Pattern Energy. The energy $\mathcal{P}$ in Eq. (14) restricted to a single triangle $i j k$ and with $u_{i j}=\log l_{i j}$ is expressed as:

$$
\begin{aligned}
& \mathcal{P}\left(u_{i j}, u_{j k}, u_{k i}\right)=\pi\left(\alpha_{i j k}\right)+\pi\left(\alpha_{j k i}\right)+\pi\left(\alpha_{k i j}\right) \\
& +u_{i j}\left(\alpha_{j k i}-\bar{\phi}_{i j}\right)+u_{j k}\left(\alpha_{k i j}-\bar{\phi}_{j k}\right)+u_{k i}\left(\alpha_{i j k}-\bar{\phi}_{k i}\right) .
\end{aligned}
$$

The Lobachevsky function $Л(\cdot)$ [Milnor 1982] and its derivative are given as:

$$
Л(x)=-\int_{0}^{x} \log (2 \sin t) d t, \quad \boldsymbol{I}^{\prime}(x)=-\log (2 \sin x) .
$$

Note that we disregarded the absolute value in $\pi$ since all the tip angles are assumed to be in the interval $(0, \pi)$. Partial derivatives of $\mathcal{P}$ are then:

$$
\begin{aligned}
& \partial_{u_{i j}} \mathcal{P}=\left(\alpha_{j k i}-\bar{\phi}_{i j}\right)+\left(u_{i j}-\log \left(2 \sin \alpha_{j k i}\right)\right) \partial_{u_{i j}} \alpha_{j k i}+ \\
& \left(u_{j k}-\log \left(2 \sin \alpha_{k i j}\right)\right) \partial_{u_{i j}} \alpha_{k i j}+\left(u_{k i}-\log \left(2 \sin \alpha_{i j k}\right)\right) \partial_{u_{i j}} \alpha_{i j k} .
\end{aligned}
$$

By applying the sine law, we have for any edge $i j$ :

$$
u_{i j}-\log \left(2 \sin \alpha_{j k i}\right)=\log \left(l_{i j} /\left(2 \sin \alpha_{j k i}\right)\right)=\log (R) .
$$

Since $\partial_{u_{i j}}\left(\alpha_{i j k}+\alpha_{j k i}+\alpha_{j k i}\right)=0$, we thus conclude:

$$
\partial_{u_{i j}} \mathcal{P}=\alpha_{j k i}-\bar{\phi}_{i j}=\left.\phi_{i j}\right|_{i j k}-\bar{\phi}_{i j} .
$$

The Hessian of $\mathcal{P}$ is now expressed as:

$$
\partial_{u_{j k}} \partial_{u_{i j}} \mathcal{P}=-\cot \alpha_{i j k}, \quad \partial_{u_{i j}} \partial_{u_{i j}} \mathcal{P}=\cot \alpha_{i j k}+\cot \alpha_{k i j} .
$$

This matrix corresponds to half of the Laplacian restricted to the triangle derived with non-conforming finite elements (see, e.g, [Polthier and Preuss 2003]), and it is therefore positive semi-definite. Consequently, the energy $\mathcal{P}$ inside $i j k$ (and thus its sum over the whole mesh) is convex within $\mathbb{L}^{\mathbb{W}}$

Capacity-Constrained Energy. The energy $\mathcal{C}$ in Eq. (16) for a single triangle $i j k$ is expressed as:

$$
\begin{aligned}
& \mathcal{C}\left(w_{i}, w_{j}, w_{k}\right)=w_{i}\left(a_{* i}-\bar{a}_{* i}\right)+w_{j}\left(a_{* j}-\bar{a}_{* j}\right)+w_{k}\left(a_{* k}-\bar{a}_{* k}\right) \\
& -\frac{1}{4}\left(\left(d_{i j}^{3}+d_{j i}^{3}\right) h_{j k i}+\left(d_{j k}^{3}+d_{k j}^{3}\right) h_{k i j}+\left(d_{k i}^{3}+d_{i k}^{3}\right) h_{i j k}\right) \\
& -\frac{1}{12}\left(l_{i j} h_{j k i}^{3}+l_{j k} h_{k i j}^{3}+l_{k i} h_{i j k}^{3}\right) .
\end{aligned}
$$

Based on Eqs. (2) and (3) and a few steps of algebra, one can show that $\partial_{w_{i}} \mathcal{C}=a_{* i}-\bar{a}_{* i}$. As $a_{* i}$ is a quadratic function of the weights (Eq. (5)), we can further deduce the Hessian of $\mathcal{C}$ as:

$$
\partial_{w_{j}} \partial_{w_{i}} \mathcal{C}=-\frac{1}{2} \frac{h_{j k i}}{l_{i j}}, \quad \partial_{w_{i}} \partial_{w_{i}} \mathcal{C}=\frac{1}{2}\left(\frac{h_{j k i}}{l_{i j}}+\frac{h_{i j k}}{l_{k i}}\right),
$$


which corresponds to one half of the Laplace-Beltrami operator $\Delta^{w}$ restricted to the triangle $i j k$. Since this operator is positive semidefinite for $(l, w) \in \mathbb{L}^{\mathbb{W}}, \mathcal{C}$ is convex.

Conformal Energy. The energy $\mathcal{E}_{c}$ in Eq. (18) in a single triangle $i j k$ of area $a_{i j k}$ is defined as:

$$
\mathcal{E}_{c}\left(l_{i j}^{2}, l_{j k}^{2}, l_{k i}^{2}\right)=\frac{1}{2}\left(\bar{\star}_{i j} l_{i j}^{2}+\bar{\star}_{j k} l_{j k}^{2}+\bar{\star}_{k i} l_{k i}^{2}\right)-a_{i j k} .
$$

By noticing that $\partial_{l_{i j}^{2}} a_{i j k}=\cot \alpha_{j k i} / 4$, one can show:

$$
\partial_{l_{i j}^{2}} \mathcal{E}_{c}=\frac{1}{2}\left(\bar{\star}_{i j}-\frac{\cot \alpha_{j k i}}{2}\right)=\frac{1}{2}\left(\bar{\star}_{i j}-\left.\star_{i j}^{\diamond}\right|_{i j k}\right) .
$$

As derived in [Zeng et al. 2012], the Hessian matrix of $\mathcal{E}_{c}$ is a positive semi-definite matrix and its elements are:

$$
\left\{\begin{array}{l}
\partial_{l_{j k}^{2}} \partial_{l_{i j}^{2}} \mathcal{E}_{c}=-R^{2} \cot \alpha_{i j k} /\left(2 l_{j k}^{2} l_{i j}^{2}\right), \\
\partial_{l_{i j}^{2}} \partial_{l_{i j}^{2}} \mathcal{E}_{c}=R^{2}\left(\cot \alpha_{i j k}+\cot \alpha_{k i j}\right) /\left(2 l_{i j}^{4}\right) .
\end{array}\right.
$$

The null-space of such a matrix contains only the vector of squared lengths $\left\{l_{i j}^{2}\right\}$.

Curvature-Constrained Conformal Energy. At last, we derive closed-form expressions for the derivatives of the energy $\mathcal{L}$ in Eq. (20) defined in a single triangle $i j k$ as:

$$
\begin{aligned}
\mathcal{L}\left(l^{2}, w\right) & =\mathcal{E}_{c}\left(l_{i j}^{2}, l_{j k}^{2}, l_{k i}^{2}\right)-w_{i}\left(\bar{\theta}_{i}-\alpha_{k i j}\right) / 2 \\
& -w_{j}\left(\bar{\theta}_{j}-\alpha_{i j k}\right) / 2-w_{k}\left(\bar{\theta}_{k}-\alpha_{j k i}\right) / 2 .
\end{aligned}
$$

Since $\mathcal{E}_{c}$ does not depend on $w$, the $w$-derivatives are:

$$
\partial_{w_{i}} \mathcal{L}=\frac{1}{2}\left(\alpha_{k i j}-\bar{\theta}_{i}\right)=\frac{1}{2}\left(\left.\theta_{i}\right|_{i j k}-\bar{\theta}_{i}\right) .
$$

For the partial derivatives w.r.t length, one has instead:

$$
\begin{aligned}
& \partial_{l_{i j}^{2}} \mathcal{L}=\frac{1}{2}\left(\bar{\star}_{i j}-\left.\star_{i j}^{\diamond}\right|_{i j k}+w_{i} \partial_{l_{i j}^{2}} \alpha_{k i j}+w_{j} \partial_{l_{i j}^{2}} \alpha_{i j k}+w_{k} \partial_{l_{i j}^{2}} \alpha_{j k i}\right) \\
& =\frac{1}{2}\left(\bar{\star}_{i j}-\frac{\cot \alpha_{j k i}}{2}-\left(w_{i}-w_{k}\right) \frac{\cot \alpha_{i j k}}{2 l_{i j}^{2}}-\left(w_{j}-w_{k}\right) \frac{\cot \alpha_{k i j}}{2 l_{i j}^{2}}\right) \\
& =\frac{1}{2}\left(\bar{\star}_{i j}-\frac{h_{j k i}}{l_{i j}}\right)=\frac{1}{2}\left(\bar{\star}_{i j}-\left.\star_{i j}\right|_{i j k}\right) .
\end{aligned}
$$

